\title{
Using Specialized Agents in a Distributed MAS to Solve Airline Operations Problems: a Case Study
}

\author{
Antonio J. M. Castro, Eugenio Oliveira \\ LIACC-NIAD\&R, Faculty of Engineering, University of Porto, Portugal \\ ajmc@fe.up.pt,eco@fe.up.pt
}

\begin{abstract}
An airline schedule very rarely operates as planned. Problems related with aircrafts, crew members and passengers are common and the actions towards the solution of these problems are usually known as operations recovery. The Airline Operations Control Center (AOCC) tries to solve these problems with the minimum cost and satisfying all the required rules. In this paper we present the implementation of a Distributed Multi-Agent System (MAS) representing the existing roles in an AOCC. This MAS has several specialized software agents that implement different algorithms, competing to find the best solution for each problem. We present a real case study where a crew recovery problem is solved. We show that it is possible to find valid solutions, in less time and with a smaller cost.
\end{abstract}

\section{Introduction}

One of the most important concerns in an airline company is the Operations Control. Through operations control mechanisms the airline company monitors all the flights checking if they follow the schedule that was previously defined by other areas of the company. Unfortunately, some problems arise during this phase [8]. Those problems are related with crew members, aircrafts and passengers. When any of these problems appear it is necessary to find solutions for them. The Airline Operations Control Centre (AOCC) is composed by teams of people specialized in solving the above problems under the supervision of an operation control manager. Each team has a specific goal contributing to the common and general goal of having the airline operation running with few problems as possible. The process of solving these problems is known as Disruption Management [7] or Operations Recovery.

Based on the observations we have done on an AOCC of a real airline company we hypothesize that the objective of solving the operations recovery problems with the less cost as possible will be much easier to achieve if we include information in the decision process related with various costs (including less easily quantifiable factors like the cost of using crew members from different operational bases) as well as if we take advantage of the fact that airlines usually have different operational bases with specific resources. Regarding crew recovery problems, we predict that if we take into account payroll information like hour salary and perdiem value of each crew rank, and costs related with hotels and extracrew travel between the different operational bases, the solution will be less expensive. The same principle can be applied to aircraft recovery and passenger recovery if we use costs related with that domain. We also hypothesize that the use of different algorithms to solve the same problem (in crew and aircraft recovery) will contribute to the robustness of the system.

In this paper we approach this problem so that it can be solved by a Multi-agent System (MAS) that represents the Operational Control Center of the airline company.

The rest of the paper is organized as follows. First we present some work of other authors regarding operations recovery. Second we introduce our proposal of a MAS for airline operations recovery, including the architecture of the MAS, the algorithm used to choose the best solution and an example of the application of our MAS. Third we present the scenario we have setup to evaluate our system as well as the results of the evaluation. Finally we discuss and conclude our work.

\section{Related work}

Traditionally, the Operations Recovery Problem has been solved through Operations Research (OR) techniques. The paper [2] gives an overview of OR applications in the air transport industry. We will present here the most recent published papers according to [6]. We divided the papers in four areas: general approaches, crew recovery, aircraft recovery and integrated recovery. For a more detailed explanation of the papers as well as for older papers related with each of these subjects, please consult [6].

General Approaches: In [7] the author's reports on the experiences obtained during the research and development of project DESCARTES (a large scale project supported by EU) on airline disruption management. The current (almost manual) mode of dealing with recovery is presented. They also present the results of the first prototype of a multiple resource decision support system.

Aircraft Recovery: The most recent paper considering the case of aircraft recovery is [11]. They formulate the problem as a Set Partitioning master problem and a route generating procedure. The goal is to minimize the cost of cancellation and retiming, and it is the responsibility of 
the controllers to define the parameters accordingly. It is included in the paper a testing using SimAir [12] simulating 500 days of operations for three fleets ranging in size from 32 to 96 aircraft servicing 139-407 flights.

Crew Recovery: In [1] the flight crew recovery problem for an airline with a hub-and-spoke network structure is addressed. The paper details and sub-divides the recovery problem into four categories: misplacement problems, rest problems, duty problems, and unassigned problems. The proposed model is an assignment model with side constraints. Due to the stepwise approach, the proposed solution is sub-optimal. Results are presented for a situation from a US airline with 18 problems.

Integrated Recovery: In [4] the author presents two models that considers aircraft and crew recovery and through the objective function focuses on passenger recovery. To test the models an AOCC simulator was developed, simulating domestic operations of a major US airline. It involves 302 aircrafts divided into 4 fleets, 74 airports and 3 hubs. Furthermore, 83869 passengers on 9925 different passengers' itineraries per day are used. For all scenarios are generated solutions with reductions in passenger delays and disruptions.

Lettovsky's Ph.D. thesis [9] is the first presentation of a truly integrated approach in the literature, although only parts of it are implemented. The thesis presents a linear mixed-integer mathematical problem that maximizes total profit to the airline while capturing availability of the three most important resources: aircraft, crew and passengers. The formulation has three parts corresponding to each of the resources, that is, crew assignment, aircraft routing and passenger flow. In a decomposition scheme these three parts are controlled by a master problem denominated the Schedule Recovery Model.

\section{A MAS for airline operations recovery}

Overview: We approached this problem by developing a distributed multi-agent system (MAS) that represents the Airline Operations Control Center (AOCC).

Each operational base has its own resources that are represented in the environment, for example, Crew Roster and Aircraft Roster are databases of schedules for the crew members and aircrafts, respectively. Other resources represented are the airport information system, legacy systems and a knowledge database for the learning capabilities of the MAS (this characteristic of the MAS will not be explained in this paper). Each operational base has also software agents that represent roles in the AOCC. The Crew Recovery Agent, Aircraft Recovery Agent and Pax Recovery Agent are dedicated to solve crew, aircraft and passengers problems, respectively, and should be seen as sub-organizations inside the MAS. The Apply Solution Agent applies the solution found and authorized in the resources of the operational base.

The MAS has also the possibility to interact with an electronic market of airline resources such as aircrafts and crew members, through the Company Broker agent. According to [7] "research on recovery operation to this date only deals with a single airline. Cooperation between airlines is not supported". With this approach we try to foster the cooperation between airlines. More information about this electronic market can be found in [10].

The MAS was developed using JADE [3] as development platform and as the run-time environment that provides the basic services for agents to execute. The MAS was developed based on a previous analysis and design by [5].

Architecture and Specialized Agents: The Crew, Aircraft and Pax Recovery agents should be seen as suborganizations. These sub-organizations have their own architecture with their specialized agents. Figure 1 shows the architecture for Crew Recovery in a UML diagram. The architecture for Aircraft Recovery and Pax Recovery are very similar.

The agent class OpMonitor is responsible for monitoring any crew events, for example, crew members that did not report for duty or duties with open positions, that is, without any crew member assigned to a specific role on board (e.g., captain or flight attendant). When an event is detected, the service MonitorCrewEvents will initiate the protocol inform-crew-event (FIPA Request) informing the OpCrewFind agent. The message will include the information necessary to characterize the event. This information is passed as a serializable object of the type CrewEvent.

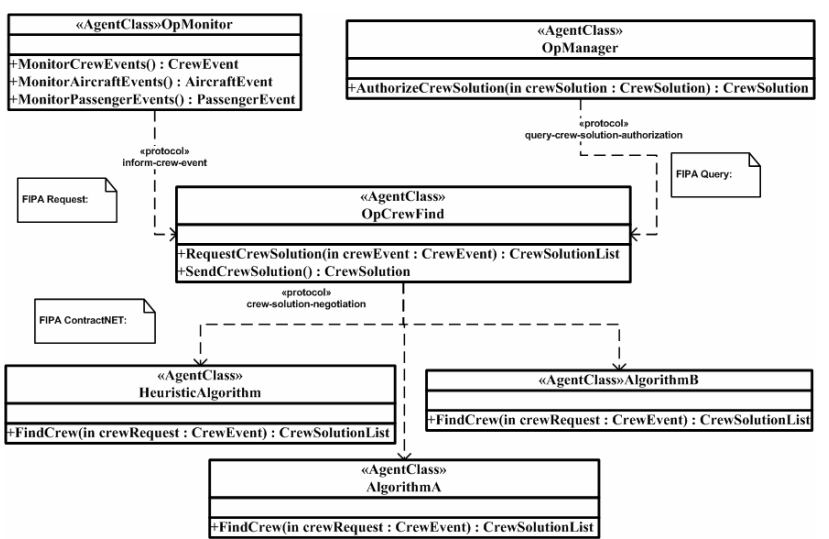

Figure 1. Crew recovery architecture

The OpCrewFind agent detects the message and will start a CFP (call for proposal) through the crew-solutionnegotiation protocol (FIPA contractNET) requesting to the specialized agents HeuristicAlgorithm, AlgorithmA and AlgorithmB (or any other that is implemented and deployed in the MAS) of any operational base of the airline company, a list of solutions for the problem. Each agent implements a different algorithm specific for this type of problem. When a solution is found a serializable object of the type CrewSolutionList is returned in the message as an answer to the CFP. 
The OpCrewFind agent collects all the proposals received and chooses the best one according to the algorithm in Table 1. This algorithm is implemented in the service SendCrewSolution and produces a list ordered by cost (a multi-criteria cost) that each solution represents. The computed values in the algorithm in Table 1 are the following:

TotalDuty: Monthly duty minutes of the proposed crew member after assigning the new duty.

CredDuty: Minutes to be paid case the crew member exceeds the monthly duty limit.

DutyPay: Cost of duty computed according to the hour salary of the crew member.

PerdiemDays: Days of work for the specific duty.

PerdiemPay: Cost of duty computed according to the perdiem value of the crew member.

BaseFactor: If the crew belongs to the same operational base where the problem happened, the value is one. Otherwise, it will have a value greater than one.

Table 1. Multi-criteria algorithm

foreach item in CrewSolution list
totalDuty $=$ monthDuty + credMins
if (totalDuty-dutyLimit $)>0$
credDuty $=$ totalDuty-dutyLimit
else
credDuty $=0$
end if
perdiemDays $=($ endDateTime-dutyDateTime
perdiemPay $=$ perdiemDays*perdiemValue
dutyPay $=$ credDuty*(hourSalaryValue/60)
cost $=($ dutyPay+perdiemPay)*baseFactor
end foreach
order all items by cost desc
select first item on the list

The first solution of the list in descendant order by cost is selected. The SendCrewSolution service initiates the protocol query-crew-solution-authorization (FIPA Query) querying the OpManager agent for authorization. The message includes the serializable object of the type CrewSolution.

\section{Scenario and experiments}

Scenario: To evaluate our MAS we have setup a scenario that includes 3 operational bases (A, B and C). Each base includes their crew members each one with a specific roster. The data used corresponded to the real operation of June 2006 of base A. We have simulated a situation where 15 crew members, with different ranks, did not report for duty in base A. The events did not happen at the same day and each one corresponds to a crew member that did not report for a specific duty in a specific day.
After setting-up the scenario we found the solutions for each crew event using two methods. In the first method we used a real user from the AOCC, with the current tools available, to find the solutions. The user uses software that shows the roster of each crew member in a Gantt chart for a specific period. The user can scroll down the information, filter according to the crew rank and base, and sort the information by name, month duty, etc. In the second method we have used our Crew Recovery Architecture and Specialized Agents of our MAS. As a final step, the solutions found by our MAS were presented to AOCC users to be validated.

Results: Table 2 presents the results that compare the two methods. From the results obtained we can see that in average, the second method took 25 seconds to find a solution and the first method took 101 seconds. Regarding the costs, the second method has a total cost of 3839 and the first method 7040. The second method is, in average 4 times faster than the first method in finding a solution and produces solutions that represent a decrease of $45.5 \%$ on the costs.

Table 2. Comparison of the results

\begin{tabular}{|l|r|r|r|r|r|}
\hline & \multicolumn{2}{|c|}{ Method 1 } & \multicolumn{2}{c|}{ Method 2 } & \multicolumn{1}{c|}{ M1/M2 } \\
\hline & Total & \multicolumn{1}{c|}{$\%$} & \multicolumn{1}{c|}{ Total } & \multicolumn{1}{c|}{ \% } & \multicolumn{1}{c|}{ \% } \\
\hline Sol. base: & & & & & \\
\hline From base A & 7 & 47 & 3 & 20,0 & $-57,1$ \\
\hline From base B & 6 & 40 & 7 & 47,0 & 16,7 \\
\hline From base C & 2 & 13 & 5 & 33,0 & 150,0 \\
\hline & & & & & \\
\hline Time (avg) & 101 & & 25 & & $-75,3$ \\
\hline Base A (avg) & 88 & 21 & 24 & 24,0 & $-72,7$ \\
\hline Base B (avg) & 110 & 27 & 24 & 24,0 & $-78,2$ \\
\hline Base C (avg) & 115 & 28 & 26 & 26,0 & $-77,4$ \\
\hline & & & & & \\
\hline Total Costs: & 7040 & & 3839 & & $-45,5$ \\
\hline Costs Base: & & & & & \\
\hline Base A & 4846 & 92,4 & 288 & 11,2 & $-94,0$ \\
\hline Base B & 1796 & 34,3 & 1276 & 49,8 & $-29,0$ \\
\hline Base C & 398 & 7,6 & 2276 & 88,8 & 472,3 \\
\hline
\end{tabular}

\section{Discussion and conclusions}

From the results we can see that our MAS obtains valid solutions faster and with less costs when compared with the current method used in a real airline company. Regarding our first hypothesis we were expecting a considerable decrease in the costs of the solutions found by our MAS. From the results obtained (see Table 2) we can see that our solution represents a decrease of $45.5 \%$ on the costs. Our hypothesis was accepted. Of course that we cannot infer that our MAS will always produce solutions that cost $45.5 \%$ less. It is not even possible to say that, in average, this decrease is valid. For that we need to evaluate much more situations, in different times 
of the year (we might have seasoned behaviors) and, then, find an average value.

From the results we can also obtain other interesting conclusions. For example, our method was $75.3 \%$ faster than method one. The use of a computerized system to find and evaluate the solutions is the reason for our method to be faster than the present, almost manual, method used in the airline. Additionally, the cooperation between different operational bases has increased with our method, because we evaluate all the solutions found (including the ones from different operational bases where the event happened) and we select the one with less cost. In method one, they choose the first one they find, usually from the same base where the event was triggered. This cooperation is also possible to be inferred from the costs by base. In Table 2 is possible to see that the costs of base $\mathrm{C}$ had an increase of $472.32 \%$ while base A and base B decreased $94 \%$ and $29 \%$, respectively. This means that our method used more resources from other bases than the base where the problem happened (base A).

Regarding our second hypothesis we expected to increase the robustness of our system using heterogeneous algorithms to find solutions to the same problem, at the same time. Preliminary results show that, most of the times, the MAS presents at least one solution even when the human operator cannot found one. Apparently this is the result of using different techniques to tackle the problem. However, we need to collect more data to analyze the impact on robustness as the result of using different specialized agents.

This paper has presented a distributed multi-agent system as a possible solution to solve airline operations recovery problems, including sub-organizations with specialized agents, dedicated to solve crew, aircraft and passenger recovery problems. We have detailed the architecture of our MAS regarding the sub-organization dedicated to solve crew recovery problems, including agents, services and protocols. We have introduced a multi-criteria algorithm for selecting the solution with less cost from those proposed as part of the negotiation process. A case study, taken from a real scenario in an airline company where we tested our method was presented and we discuss the results obtained. We have shown that our method produces faster and less expensive solutions when compared with the present and almost manual method, used in the airline company.

Further work is required in testing our method for large periods of time and in different times of the year (due to seasoned behaviors). We also need to test our MAS with all the sub-organizations working at the same time (crew, aircraft and passenger) to see the impact that might exist in the results we have presented in this paper. Finally, we would like to apply and test the integration of the EM as presented in [10].

\section{References}

[1] Abdelgahny, A., Ekollu, G., Narisimhan, R., and Abdelgahny, K. 2004. A Proactive Crew Recovery Decision Support Tool for Commercial Airlines during Irregular Operations. Annals of Operations Research 127:309-331.

[2] Barnhart, C., Belobaba, P., and Odoni, A. 2003. Applications of Operations Research in the Air Transport Industry. Transportation Science 37:368-391.

[3] Bellifemine, F., Caire, G., Trucco, T., and Rimassa, G. 2004. JADE Programmer's Guide. JADE 3.3 TILab S.p.A.

[4] Bratu, S., and Barnhart, C. 2006. Flight Operations Recovery: New Approaches Considering Passenger Recovery. Journal of Scheduling 9(3):279-298.

[5] Castro, A., and Oliveira, E. 2005. A Multi-Agent System for Intelligent Monitoring of Airline Operations. In Proceedings of the Third European Workshop on Multi-Agent Systems, 91102. Brussels, Belgium: EUMAS 2005.

[6] Clausen, J., Larsen, A., and Larsen, J. 2005. Disruption Management in the Airline Industry - Concepts, Models and Methods. Technical Report, 2005-01, Informatics and Mathematical Modeling, Technical University of Denmark, DTU.

[7] Kohl, N., Larsen, A., Larsen, J., Ross, A., and Tiourline, S. 2004. Airline Disruption Management - Perspectives, Experiences and Outlook. Technical Report, CRTR-0407, Carmen Research.

[8] Kohl, N., and Karish, S. 2004. Airline Crew Rostering: Problem Types, Modeling and Optimization, Annals of Operations Research 127: 223-257.

[9] Lettovsky, L. 1997. Airline Operations Recovery: An Optimization Approach. Ph.D. diss., Georgia Institute of Technology, Atlanta, USA.

[10] Malucelli, A., Castro, A., and Oliveira, E. 2006. Crew and Aircraft Recovery Through a Multi-Agent Electronic Market. In Proceedings of IADIS International Conference eCommerce, 51-58. Barcelona, Spain: IADIS Press, ISBN: 9728924-23-2.

[11] Rosenberger, J., Johnson, E., and Nemhauser, G. 2001. Rerouting aircraft for airline recovery. Technical Report, TLILEC 01-04, Georgia Institute of Technology.

[12] Rosenberger, J., Schaefer, A., Goldsmans, D., Johnson, E., Kleywegt, A., and Nemhauser, G. 2002. A Stochastic Model of Airline Operations, Transportation Science 36(4):357-377. 